\title{
Pyruvate kinase is a dosage-dependent regulator of cellular amino acid homeostasis
}

\author{
Katharina Bluemlein ${ }^{1, *}$, Matthias Glückmann ${ }^{2, *}$, Nana-Maria Grüning ${ }^{1}$, René \\ Feichtinger ${ }^{4}$, Antje Krüger ${ }^{3}$, Mirjam Wamelink ${ }^{6}$, Hans Lehrach ${ }^{3}$, Stephen Tate ${ }^{5}$, \\ Daniel Neureiter7, Barbara Kofler ${ }^{4}$, and Markus Ralser ${ }^{1,3, *}$ \\ ${ }^{1}$ Department of Biochemistry \& Cambridge Systems Biology Centre, University of Cambridge, Cambridge, United Kingdom \\ ${ }^{2}$ AB SCIEX, Landwehrstrasse, Darmstadt, Germany \\ ${ }^{3}$ Max Planck Institute for Molecular Genetics, Berlin, Germany \\ ${ }^{4}$ Research Program for Receptor Biochemistry and Tumor Metabolism, Department of Pediatrics, Paracelsus Medical \\ University, University Hospital Salzburg, Salzburg, Austria \\ ${ }^{5}$ AB SCIEX, Concord, Ontario, Canada \\ ${ }^{6}$ VU University Medical Center Amsterdam , Amsterdam, The Netherlands \\ ${ }^{7}$ Institute of Pathology, Paracelsus Medical University, University Hospital Salzburg, Salzburg, Austria \\ * Denotes equal contribution
}

Correspondence to: Markus Ralser, email: mr559@cam.ac.uk

Keywords: cancer metabolism, pyruvate kinase, proteomics, amino acid profile

Received: October 29, 2012, Accepted: October 30, 2012, Published: November 01, 2012

Copyright: ( B Bluemlein et al. This is an open-access article distributed under the terms of the Creative Commons Attribution License, which permits unrestricted use, distribution, and reproduction in any medium, provided the original author and source are credited.

ABSTRACT:

The glycolytic enzyme pyruvate kinase (PK) is required for cancer development, and has been implicated in the metabolic transition from oxidative to fermentative metabolism, the Warburg effect. However, the global metabolic response that follows changes in PK activity is not yet fully understood. Using shotgun proteomics, we identified 31 yeast proteins that were regulated in a PK-dependent manner. Selective reaction monitoring confirmed that their expression was dependent on PK isoform, level and activity. Most of the PK targets were amino acid metabolizing enzymes or factors of protein translation, indicating that PK plays a global regulatory role in biosynthethic amino acid metabolism. Indeed, we found strongly altered amino acid profiles when PK levels were changed. Low PK levels increased the cellular glutamine and glutamate concentrations, but decreased the levels of seven amino acids including serine and histidine. To test for evolutionary conservation of this PK function, we quantified orthologues of the identified PK targets in thyroid follicular adenoma, a tumor characterized by high PK levels and low respiratory activity. Aminopeptidase AAP-1 and serine hydroxymethyltransferase SHMT1 both showed PKM2- concentration dependence, and were upregulated in the tumor. Thus, PK expression levels and activity were important for maintaining cellular amino acid homeostasis. Mediating between energy production, ROS clearance and amino acid biosynthesis, PK thus plays a central regulatory role in the metabolism of proliferating cells.

\section{INTRODUCTION}

Cancer cell growth is characterized by reconfiguration of cellular metabolism. Different routes of intermediary and biosynthetic metabolism, including nucleic acid synthesis, amino acid biosynthesis, and energy production are reconfigured [1-4]. A characteristic feature of many tumors is an augmentation of nonoxidative (fermentative) metabolism at the expense of oxidative metabolism (respiration), although the latter would be more efficient for energy production. Known as the 'Warburg effect', this metabolic reconfiguration is considered as a 'hallmark' of cancer, and may have high therapeutic potential $[5,6]$. 
Recent results indicate that the Warburg effect could be the result of metabolic balancing processes which avoid the accumulation of toxic reactive oxygen species (ROS) maintaining metabolic homeostasis [7, 8]. Indeed, cancer cells suffer from high loads of ROS which may make them vulnerable in pro-oxidant therapies [8-10]. ROS originate from biochemical reactions, i.e. the degradation of fatty acids, and leakage from complex I and III of the respiratory chain $[11,12]$. Clearing of these reactive molecules prevents oxidative stress, and is conducted by large machinery which involves central carbon metabolism as essential metabolic component. Fluxes of glycolysis and the adjacent pentose phosphate pathway (PPP) are adjusted when cells encounter an oxidative environment [13]. This metabolic reconfiguration fuels the antioxidative machinery with its main redox cofactor NADPH, required by the glutathione, peroxiredoxin and thioredoxin systems $[14,15]$, and is involved in regulating gene expression during oxidative conditions [16].

The metabolic enzyme pyruvate kinase $(\mathrm{PK})$ is required for coordination of these pathways when cells encounter increased ROS levels. Lowering PK activity in yeast and human increases resistance to oxidants $[17$, 18]. At least in part, this adaptation is mediated through accumulation of the PK substrate phosphoenolpyruvate. PEP causes feedback inhibition of several glycolytic enzymes, including triosephosphate isomerase (TPI) [17]. Lowered TPI activity increases oxidant resistance as studied in yeast and C. elegans, and acts through redirecting the metabolic flux from glycolysis into the PPP. This increases the $\mathrm{NADP}^{+}$reduction rate [13], and triggers an adaptation of gene expression towards oxidative conditions [16].

In this study, we focused on the regulatory events upstream and downstream of PK, picturing PK induced proteome changes in yeast and human. For this, we combined workflows of quantitative proteomics which circumvent the use of isotope labeled standards ('labelfree proteomics'). In yeast, low levels of PK were sufficient to induce changes in the biosynthetic proteome, primarily affecting protein biosynthesis and amino acid metabolism. Subsequently, ion exchange chromatography was used to profile free amino acid levels in yeast strains possessing different PK activities. Low PK activity caused a decline in the concentration of arginine, aspartic acid, histidine, lysine, serine, threonine, and valine, and an increase in glutamine and glutamate, thus triggered a broad reconfiguration of the yeast amino acid profile.

In human thyroid follicular adenoma, we observed upregulation of two orthologues of the identified yeast proteins, aminopeptidase AAP-1 and serine hydroxymethyltransferase SHMT1, and provide evidence that their expression level might quantitatively correlate with PKM2 expression in the cancer state. Thus, in yeast and humans, PK emerges as regulator of carbohydrate, energy and also amino acid metabolism.

\section{RESULTS}

\section{PK levels target cellular biosynthetic metabolism}

Although of reduced catalytic activity [18, 19], the expression level of the predominant human pyruvate kinase isoform PKM2 is increased in cancers [20]. In yeast, changes in PK isoform and expression levels are observed when cells switch from fermentation to oxidative metabolism $[17,21]$, indicting that PK plays an evolutionary conserved role in the underlining metabolic reconfiguration.

Previously, we have shown that lowering PK activity in yeast increases the rate of respiration, and at the same time augments oxidant resistance [17]. This indicates this enzyme is required for coordination of respiration and ROS clearance. Here, we used mass spectrometry based proteomics to picture the global physiological consequences triggered by a change in PK levels. We compared two engineered yeast strains that just differ in the promoter $\left(T E F 1_{p r}\right.$ for high level expression and $C Y C 1_{p r}$ for low level expression) used to express yeast Pyk1p, the high active yeast PK isoform [17, 21]. MS/ MS shotgun proteome profiles of these two yeast strains were recorded using a nanoLC (Ultimate 3000 (Dionex)) coupled QqTOF mass spectrometer TripleTOF5600 (AB/Sciex) [22]. Obtained MS/MS spectra were used to identify peptides and proteins by a database search with ProteinPilot ${ }^{\mathrm{TM}}$ software (AB SCIEX). A typical run yielded the identification of 922 proteins at global FDR of $<1 \%$ from the UniprotKB/Swiss-Prot database 57.15. At a global FDR of $1 \%$ on the peptide level 6393 peptides and on spectrum level 72256 spectra were identified.

The identified proteins and peptides were analyzed with PeakView ${ }^{\mathrm{TM}}$ software (AB SCIEX), where mass and extracted ion chromatograms (XICs) were obtained for the confidentially identified peptides (Peptide ID with unique peptides at a global false discovery rate $<$ $1 \%$ ). The same peptides were extracted for all technical and biological replicates. The extraction window for all peptide XICs was set to $0.01 \mathrm{Da}$ to increase specificity and improve the quantification results. Typical elution profiles for peptides show a width of 20 seconds (FWHM) at the HPLC conditions selected to ensure a number of $>$ 8 points for quantification, typically at $<35$ points across peak base. Extracted ion chromatograms of the tryptic Pyk $1 p$ peptide K.TNNPETLVAL.R of cells with different PYK1 expression levels, 19 data points per peak, illustrate clear separation between high (A) and low (B) level expression of PK in three replicates each (Fig 1A). For relative quantification, sum of the total peak area was used to normalize peak areas between the different samples. Fig 1B illustrates the normalized peak area for all identified Pyk1p-belonging peptides over technical and biological replicates. In total, $42.6 \%$ of identified proteins met the 

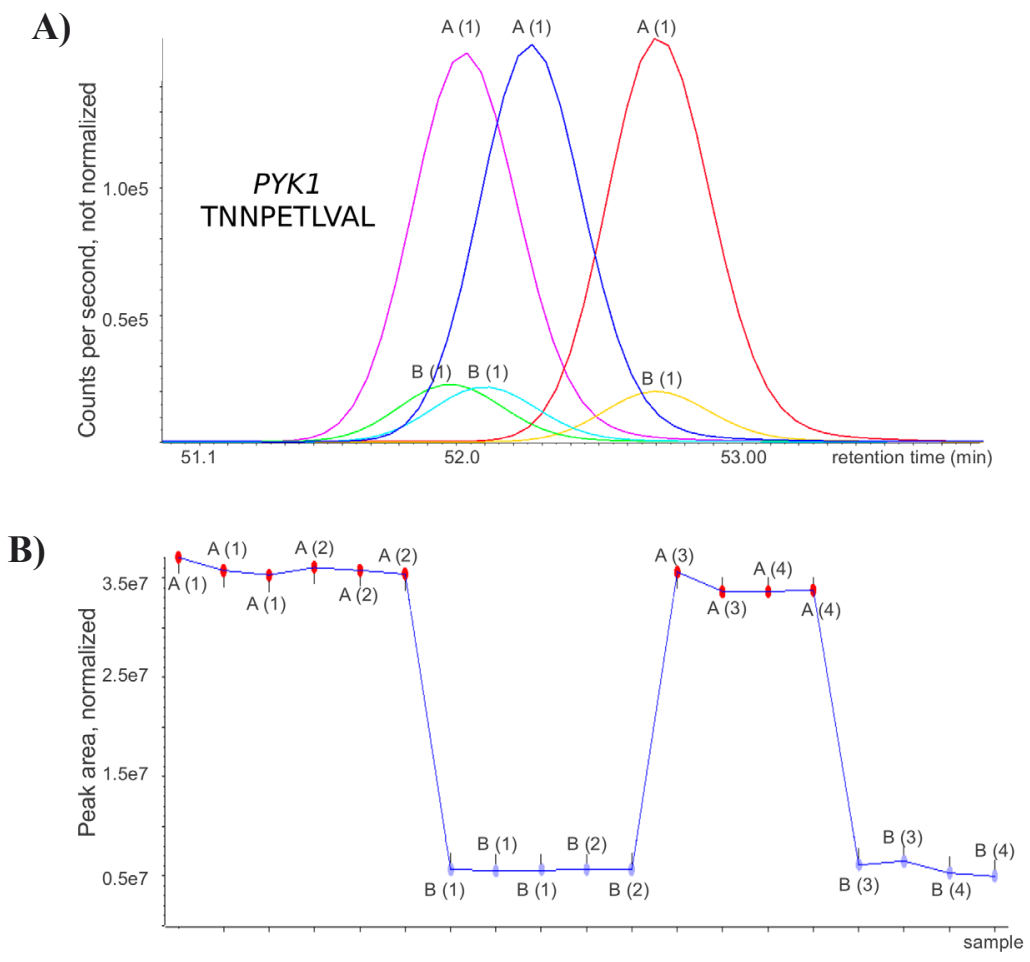

C)

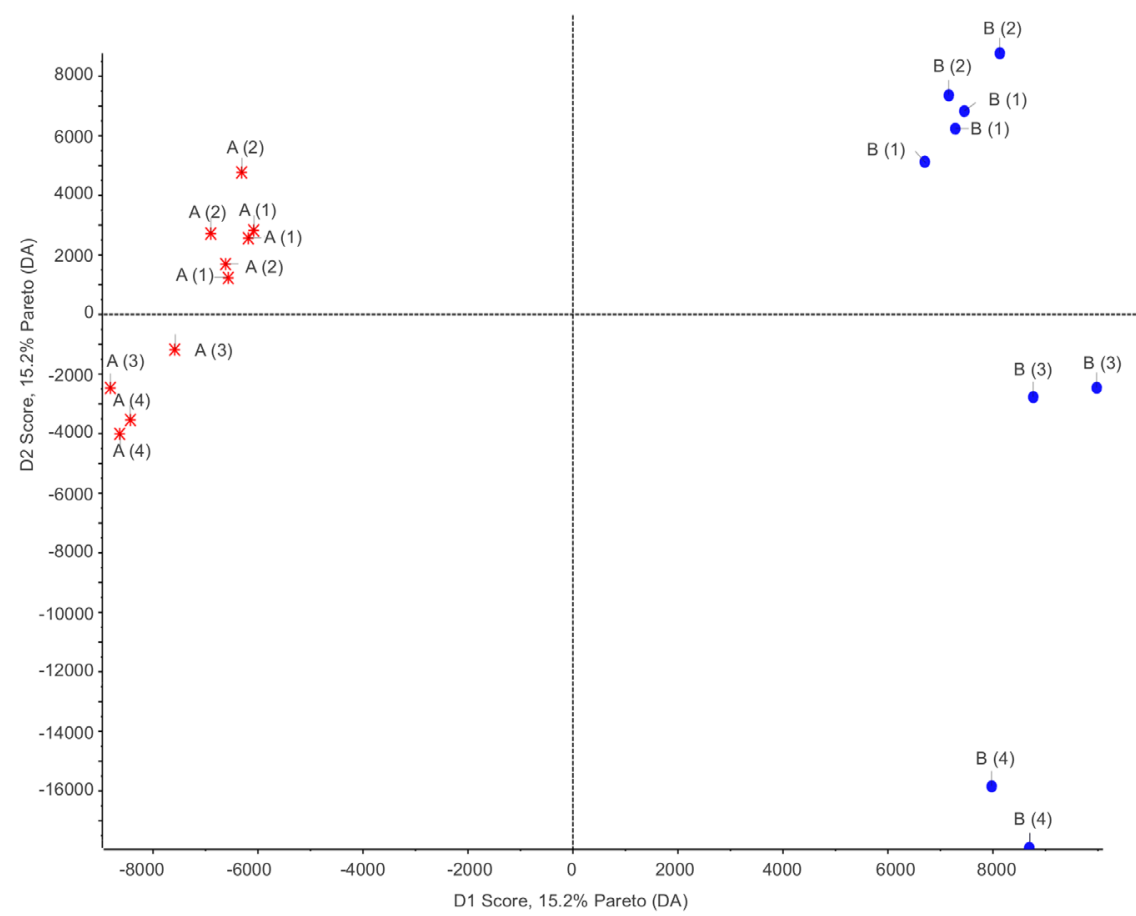

Figure 1: Label-free shotgun proteome profiles of yeast expressing Pyk1p at high and low level. (A)Tryptic digests generated from yeast protein extracts were analyzed on an AB SCIEX QqTOF mass spectrometer. Extracted ion chromatograms for the Pyklp tryptic peptide K.TNNPETLVAL.R from three LC-MS/MS shotgun runs each of yeast expressing Pyk1p at high level (,A“ samples) or low level (,B‘ samples).(B)Peak area of all Pyk1p-associated peptides in cells expressing Pyk1p at and high and low level, normalized to the total peak area of the respective run. Replicate injections of the same sample (LC-MS technical replicates) are marked with the same label; Numbers (1) \& (2), respectively (3) \& (4) indicate sample preparations and tryptic digests from independently grown yeast cultures. (C)Principal component analysis after pareto scaling of the shotgun proteome profiles, generated with MarkerView ( $\mathrm{AB} / \mathrm{Sciex})$. A change in the Pyklp expression level clearly separates the proteomes. Determined by two tailed T-test, 31 proteins (Table 1) were significantly ( $p$ $<0.05)$ regulated and responsible for the separation of the proteome profiles. 
Upregulated (TEFpr-PYK1 compared to CYCpr-PYK1)

\begin{tabular}{|c|c|c|c|c|c|}
\hline \multicolumn{3}{|c|}{ Row } & t-value & \multirow{2}{*}{$\begin{array}{l}\mathrm{p} \text {-value } \\
1.33 \mathrm{E}-22 \\
\end{array}$} & \multirow{2}{*}{$\begin{array}{l}\text { Foldchange } \\
6.287671\end{array}$} \\
\hline 1 & P00549 & KPYK1_YEAST & 72.27618 & & \\
\hline 2 & P00815 & HIS2_YEAST & 15.3411 & $2.17 \mathrm{E}-11$ & 2.445479 \\
\hline 3 & P09436 & SYIC_YEAST & 13.50707 & $1.61 \mathrm{E}-10$ & 1.729696 \\
\hline 4 & P22943 & HSP12_YEAST & 12.0193 & $9.82 \mathrm{E}-10$ & 1.674836 \\
\hline 5 & P37898 & AAP1_YEAST & 9.55664 & $3.00 \mathrm{E}-08$ & 4.608135 \\
\hline 8 & P05750 & RS3_YEAST & 8.541646 & $1.48 \mathrm{E}-07$ & 1.249022 \\
\hline 10 & P15108 & HSC82_YEAST & 8.178904 & 2.70E-07 & 1.291876 \\
\hline 12 & Q07478 & SUB2_YEAST & 7.612269 & 7.13E-07 & 1.446553 \\
\hline 13 & P05694 & METE_YEAST & 7.49884 & $8.71 \mathrm{E}-07$ & 1.289915 \\
\hline 16 & P20606 & SAR1_YEAST & 6.706529 & 3.69E-06 & 4.203254 \\
\hline 17 & P05319 & RLA2_YEAST & 5.961237 & $1.55 \mathrm{E}-05$ & 1.204707 \\
\hline 19 & P05755 & RS9B_YEAST & 5.801303 & $2.13 \mathrm{E}-05$ & 1.172176 \\
\hline 22 & P37292 & GLYM_YEAST & 5.658496 & 2.83E-05 & 1.264169 \\
\hline 23 & P31539 & HS104_YEAST & 5.619102 & $3.07 \mathrm{E}-05$ & 1.279804 \\
\hline 25 & P19358 & METK2_YEAST & 5.567696 & $3.40 \mathrm{E}-05$ & 1.388585 \\
\hline 26 & P19882 & HSP60_YEAST & 5.533043 & $3.65 \mathrm{E}-05$ & 1.177094 \\
\hline 27 & P49089 & ASNS1_YEAST & 5.473253 & $4.12 \mathrm{E}-05$ & 1.361167 \\
\hline 28 & P38011 & GBLP_YEAST & 5.441745 & 4.40E-05 & 1.325665 \\
\hline 29 & P48164 & RS7B_YEAST & 5.351547 & $5.29 \mathrm{E}-05$ & 1.221251 \\
\hline 30 & P41940 & MPG1_YEAST & 5.328021 & 5.55E-05 & 1.420641 \\
\hline 32 & P27476 & NSR1_YEAST & 5.180612 & $7.52 \mathrm{E}-05$ & 1.511833 \\
\hline
\end{tabular}

Downregulated (TEFpr-PYK1 compared to CYCpr-PYK1)

Row
\begin{tabular}{|l|l|l|l|l|l|l|}
\hline 6 & P00924 & ENO1_YEAST & -9.00757 & & $7.00 \mathrm{E}-08$ & 0.577695 \\
\hline 7 & P38720 & 6PGD1_YEAST & -8.83961 & & $9.14 \mathrm{E}-08$ & 0.70217 \\
\hline 9 & P00358 & G3P2_YEAST & -8.21728 & & $2.53 \mathrm{E}-07$ & 0.403518 \\
\hline 11 & Q00055 & GPD1_YEAST & -7.84349 & & $4.77 \mathrm{E}-07$ & 0.689863 \\
\hline 14 & P00830 & ATPB_YEAST & -7.24239 & & $1.38 \mathrm{E}-06$ & 0.789716 \\
\hline 15 & P41277 & GPP1_YEAST & -6.71021 & & $3.66 \mathrm{E}-06$ & 0.664699 \\
\hline 18 & P00950 & PMG1_YEAST & -5.90746 & & $1.72 \mathrm{E}-05$ & 0.757085 \\
\hline 20 & Q05911 & PUR8_YEAST & -5.76513 & & $2.29 \mathrm{E}-05$ & 0.720522 \\
\hline 21 & Q07551 & KAR_YEAST & -5.71729 & & $2.52 \mathrm{E}-05$ & 0.652231 \\
\hline 24 & P38972 & PUR4_YEAST & -5.61894 & & $3.07 \mathrm{E}-05$ & 0.823931 \\
\hline 31 & P00925 & ENO2_YEAST & -5.21596 & & $6.99 \mathrm{E}-05$ & 0.828283 \\
\hline
\end{tabular}

stringent criteria of being identified with $>3$ unique peptides in all 19 data-dependent acquisition runs, thus were used for quantification.

\section{PK targets respond to level changes of both yeast PYK isozymes}

We first analyzed the shotgun proteome profiles by principal component analysis (PCA) after pareto sorting. Profiles obtained from both from technical and biological replicates showed clear clustering according to whether Pyk1 was expressed at high or low level (Fig 1C). Thus, 
a change in the expression level of the Pyk1p enzyme caused a reproducible change in the yeast proteome.

We continued our investigations by an in depth verification of the proteomic results making use of a quantitative MS technique, selective reaction monitoring (SRM). In difference to shotgun proteomics, SRM assays are generated in a targeted way and on the base of the protein/peptide sequence or its known fragmentation spectrum [23]. With SRM, a much lower number of molecules per sample can be quantified (here we choose nine proteins randomly), but these assays are more sensitive due to improved signal to noise ratio, therefore facilitating faster runtimes, and are more reproducible, and are thus used in diagnostics [23-25]. We established and validated SRM assays for a representative number of proteins which were significantly $(\mathrm{p}<0.05)$ responsible for separating the proteomes in the PCA (Fig 1C, Table
1). The SRM assays were applied to BY4741, the two Pyk1p strains used in the QqTOF analysis (Fig 1A), and two further yeast strains $\left(T E F_{p r}-P y k 2\right.$ and $\left.C Y C_{p r}-P y k 2\right)$ which express the low active Pyk2 isozyme at high and low level (please see Fig 2A for an overview, strains were in detail described in [17])

Label-free SRM confirmed the PYK-dependent regulation as identified with the shotgun platform for 7 of the 9 proteins (Fig 2, Suppl. Table 1 for numeric values). Interestingly, all Pyk1p regulated proteins were also dependent on the Pyk $2 p$ expression level, thus they were not regulated in an isoform specific manner. Remarkably, we observed that the expression pattern grouped the PK targets according to their biological function. The first group of co-regulated proteins was formed by the glycolytic enzymes glycerol-3-phosphatase dehydrogenase, phosphoglycerate mutase, and enolase 2.
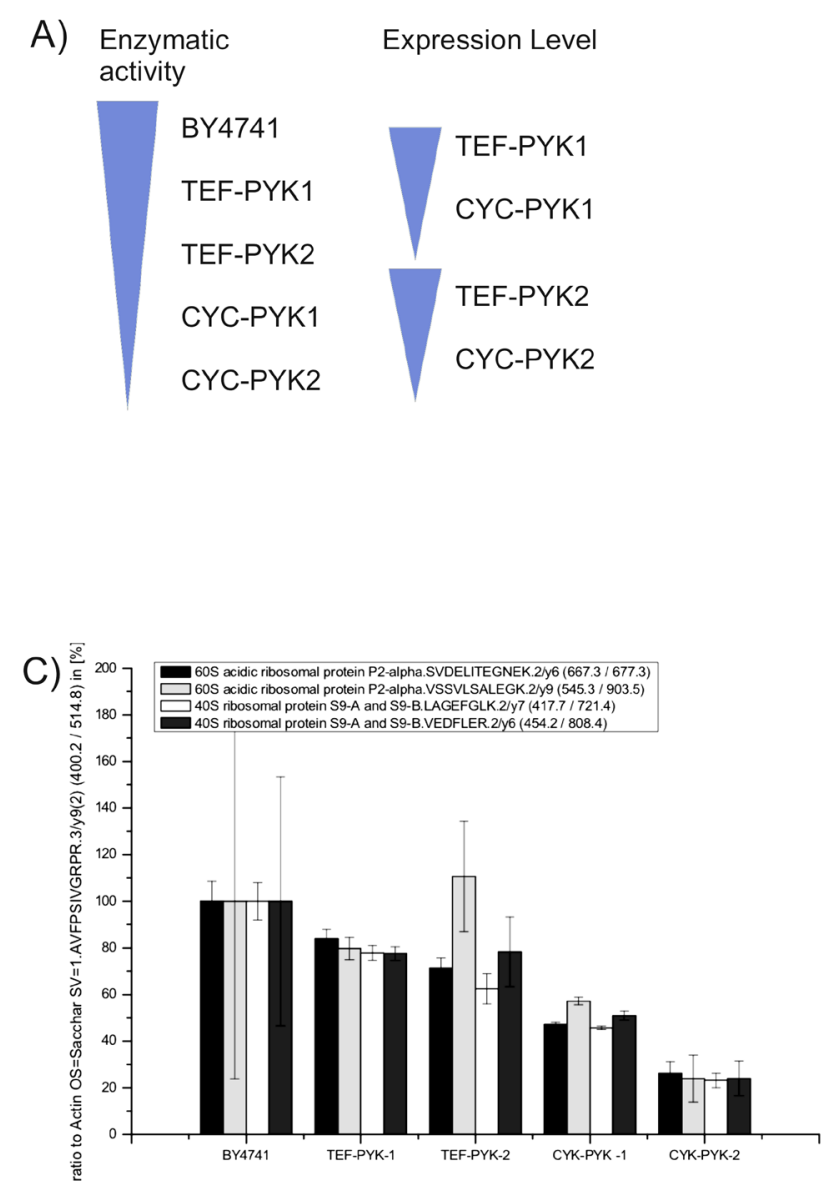

B)
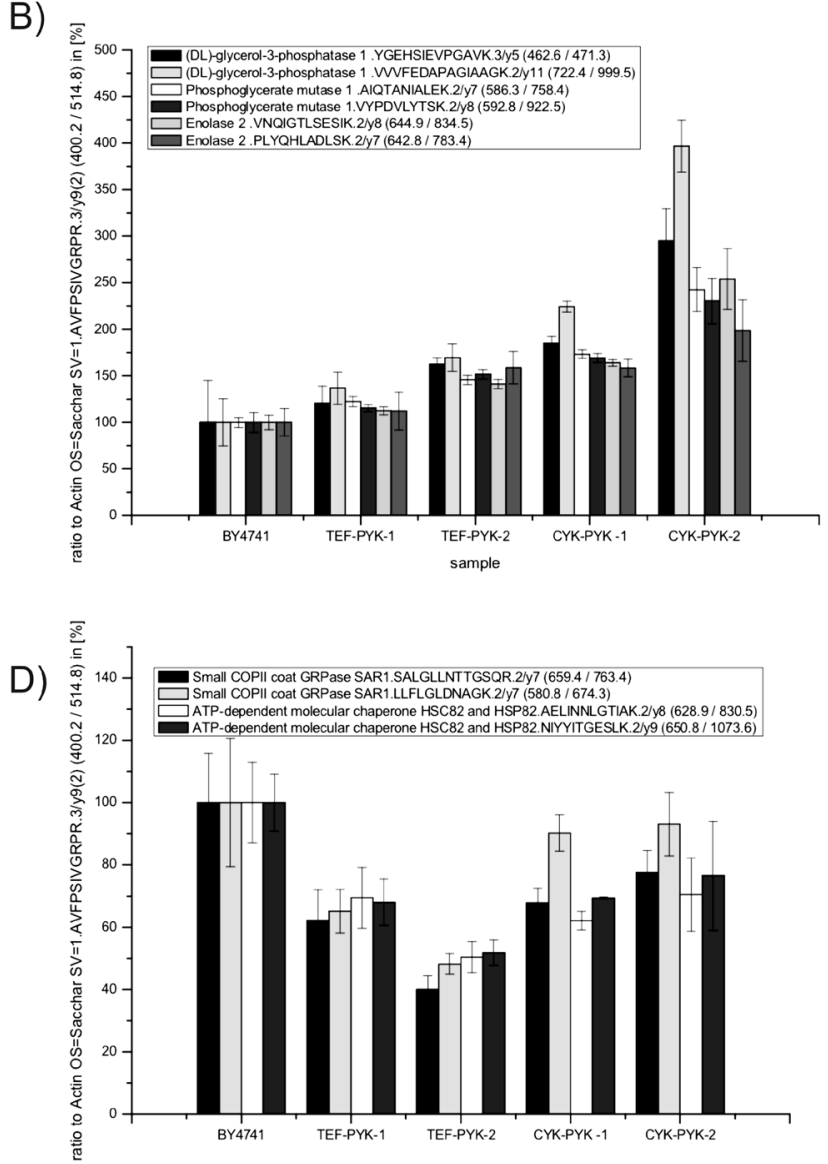

Figure 2: Targeted quantification of PK regulated genes by selected reaction monitoring. (A)Overview of the yeast model strains [17] that differ in overall PYK activity, Pyk isoform, and expression level. (Left bars) Total PK activity changes over BY4741 > TEFpr-PYK1 > TEFpr-PYK2 > CYCpr-PYK1 to CYCpr-PYK2, as described previously [17] . (Right bars). High expression of Pyk1p and Pyk $2 \mathrm{p}$ under control of the TEF1 promoter, low expression under control of the CYC1 promoter [17]. (B)Glycolytic enzymes glycerol-3-phosphatase, phosphoglycerate mutase 1, and enolase 2 are upregulated in yeast strains with lower PK activity. (C)Ribosomal proteins quantified using single reaction monitoring (60S acidic ribosomal protein P2-alpha and 40S ribosomal protein S9-A and S9-B) are downregulated in yeast strains with lower PK activity. (D)PYK targets Sar1 and Hsp82 are regulated in strains with altered PYK1 expression, but are not correlating with the overall enzyme activity. (A-D) Determined expression values are given in the Suppl. Table 1. 


\section{A)}

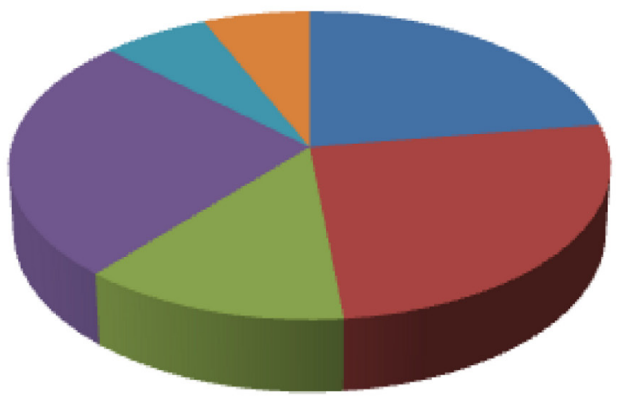

Amino acid \& secondary metabolism

- Translation \& ribosome biogenesis

- Chaperones

- Glycolysis \& pentose phosphate pathway

n Nucleotide biosynthesis

- Other

\begin{tabular}{|c|c|c|c|c|c|}
\hline Gene & Function & Acc. No & $\begin{array}{c}p- \\
\text { value }\end{array}$ & $\begin{array}{l}\text { Human } \\
\text { orthologue }\end{array}$ & Acc. No \\
\hline$A A P 1$ & Arginine/alanine aminopeptidase & P37898 & $3.00 \mathrm{E}-008$ & AAP-S (PSA1) & P55786 \\
\hline SHM1 & Serine hydroxymethyltransferase & P37292 & 2.83E-005 & SHMT1 & NP_004160 \\
\hline PSA1 & GDP-mannose pyrophosphorylase & P41940 & $5.55 \mathrm{E}-005$ & GMPPB & $\begin{array}{l}\text { NP_037466. } \\
2\end{array}$ \\
\hline ASN1 & Asparagine synthetase & P49089 & 4.12E-005 & ASNS & P08243 \\
\hline SAM2 & S-adenosylmethionine synthetase & P19358 & $3.40 \mathrm{E}-005$ & METK1 & Q00266 \\
\hline HIS4 & Histidine bio synthesis & $\mathrm{P} 00815$ & 2.17E-011 & & \\
\hline MET6 & Methionine synthase & P05694 & 8.71E-007 & & \\
\hline
\end{tabular}

B) amino acids, decrease in concentration

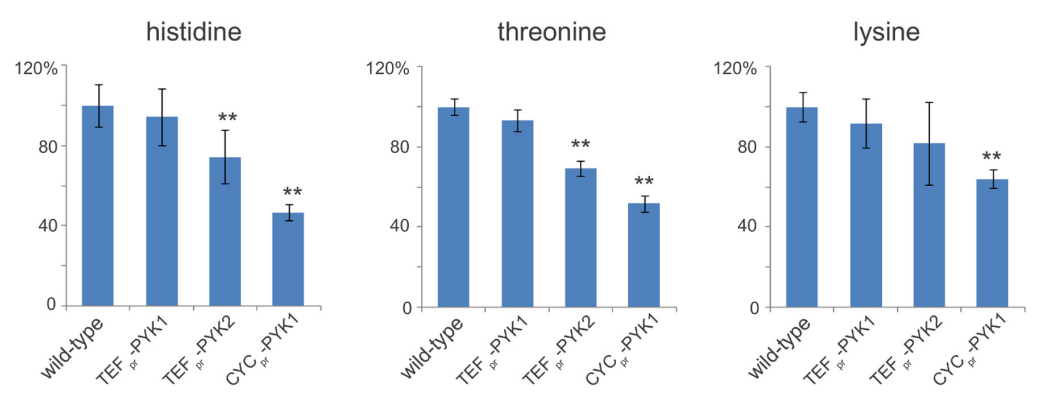

C) amino acids, increase in concentration

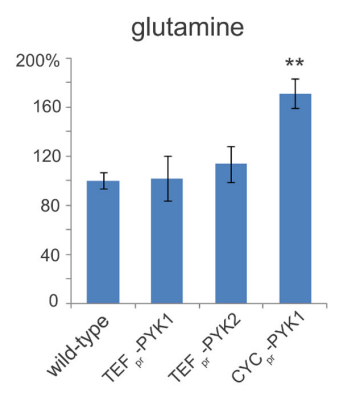

glutamate

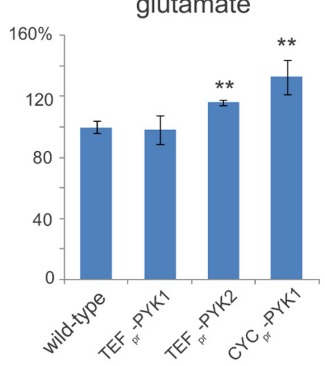

D)

\begin{tabular}{ll}
\multicolumn{1}{|c|}{$\begin{array}{l}\text { Significantly altered amino acids } \\
(p<0.05)\end{array}$} \\
\hline decreased \\
Arginine & Aspartic acid \\
\hline Histidine & Lysine \\
\hline Serine & Threonine \\
\hline $\begin{array}{l}\text { Valine } \\
\text { increased }\end{array}$ \\
\hline Glutamine & Glutamic acid \\
\hline
\end{tabular}

Figure 3: Amino acid and biosynthethic metabolism are targets of yeast PK. (A)PK targets identified in by proteome profiling belong to primary metabolism as well to amino acid- and protein biosynthesis (upper panel). The table lists the genes which play a role in amino acid and protein biosynthethic metabolism, and their direct human orthologue (if applicable) (B)Pyruvate kinase affects the concentration of nine amino acids. Illustrated is the concentration of histidine, threonine and lysine (decreased) or (C)Glutamine, glutamate (increased) relative to their level in BY4741 (wild-type). Significant ( $<<0.05$, two-tailed T test) concentration changes are marked $(* *)$. (D) In total, nine out of 17 amino acids were found to be significantly regulated ( $p<0.05$, two-tailed $\mathrm{T}$ test), by PK activity; these amino acids except glutamine and glutamate were present in lower concentrations in yeast with low PK activity. 
All these proteins were gradually upregulated as lower overall activity of PK was (Fig 2B). Second, ribosomal proteins grouped together, and showed the exact opposite trend being down-regulated in strains with lower PK activity (Fig 2C). Finally, there was the third group of proteins which was clearly affected by altered PYK expression, but did not correlate with the enzymatic activity (Fig 2D). This group was represented by $S A R 1$, a small COPII coat GTPase and HSC82/ HSP82, an ATPdependent molecular chaperone. These proteins were expressed at quite similar levels in TEF-PYK1 and TEF$P Y K 2$ yeast, at higher levels in $C Y C-P Y K 1$ and $C Y C$ $P Y K 2$ yeast, and even higher levels in the wild-type strain BY4741(Fig 2D).

Hence, low PK activity led to an up-regulation of glycolytic proteins assayed, and at the same time to a downregulation of tested components of the translational machinery. Furthermore, the proteome study identified PK target proteins which responded in an activity independent manner. Finally, all proteins found to be regulated by $P Y K 1$ were also controlled by $P Y K 2$, Thus, both expression level and enzymatic activity of PK show to have an effect on protein expression regulation.

\section{PK expression levels affect the concentration of free amino acids}

Virtually all proteins responsible for the clear division of the PYK proteomes were involved in energy,

Thyroid Follicular Adenoma
A) PKM1 and PKM2 levels

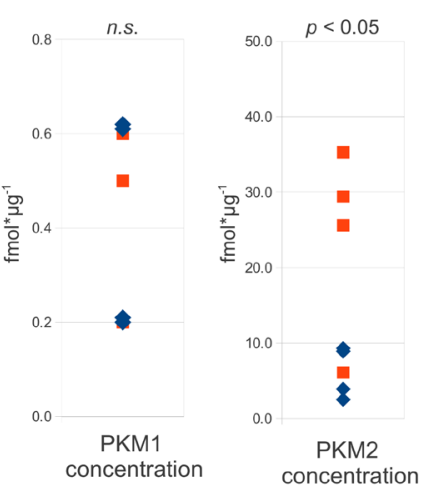

B) Activity of respiratory chain complexes I - V

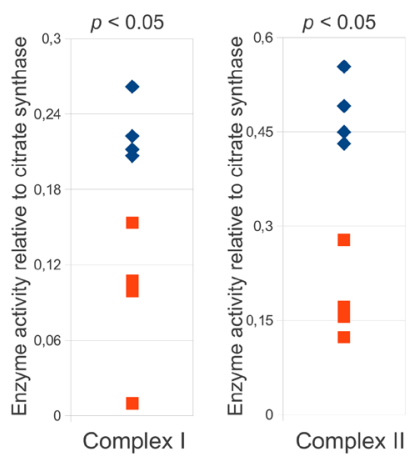

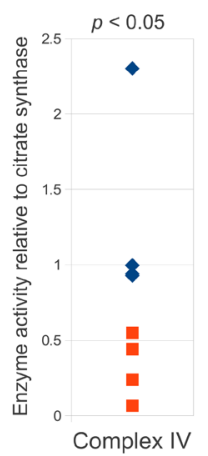
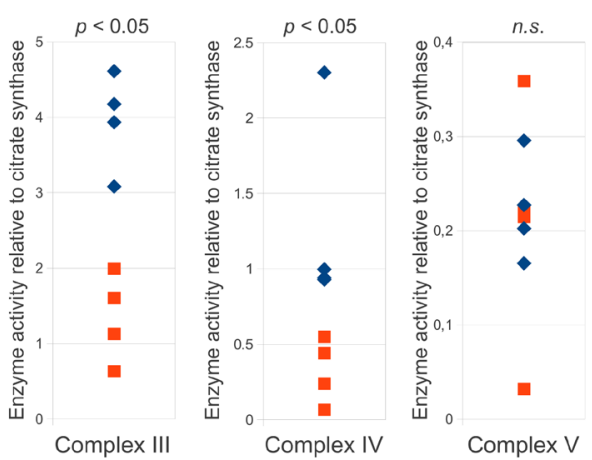

Complex V
C) aminopeptidase, puromycin sensitive (AAP-S)

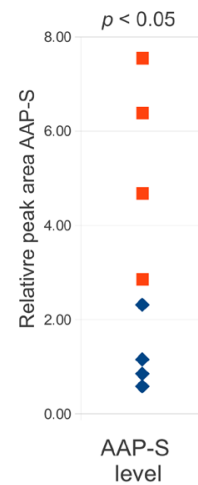

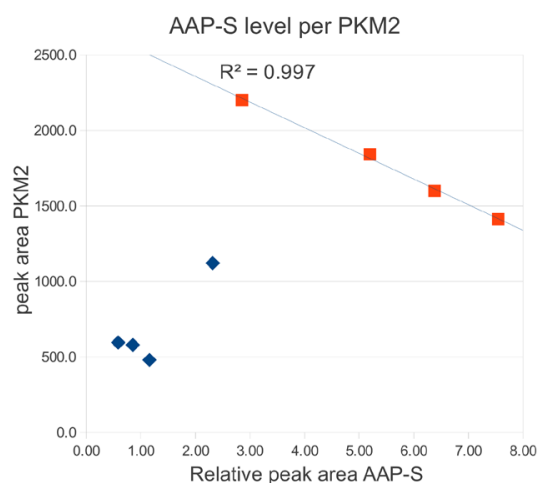

D) Serine hydroxymethyltransferase 1 (SHMT1)

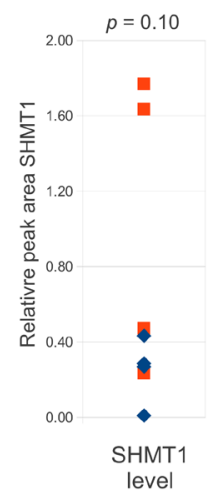

Figure 4: AAP-S and SHMT1 are upregulated in thyroid follicular adenoma and correlate with the PKM2 expression level. (A)PKM2, but not PKM1 concentration, distinguishes thyroid follicular adenoma from normal thyroid. PKM1 and PKM2 were quantified in tumor and the controls [20], the absolute PKM concentrations in individual biopsies are plotted. (B)Activity of the respiratory chain is reduced in thyroid follicular adenoma. Enzyme activity of complexes I - V of the four tumors and control tissues relative to the activity of citrate synthase. (C)Puromycin sensitive aminopeptidase (AAP-S) is upregulated in thyroid follicular adenoma, and correlates with PKM2 in the tumor state. Relative level of AAP-S in tumor and normal thyroid (left panel), AAP-S expression level plotted versus the PKM2 concentration in the same biopsy (right panel). (D)Serine hydroxymethyltransferase 1 (SHMT1) shows clear trends towards higher expression in thyroid follicular adenoma, and seem to correlate with PKM2 in the tumor state. Relative level of SHMT1in tumor and normal thyroid (left panel), SHMT1expression level plotted versus the PKM2 concentration (right panel). (A-D) p-values were determined with two-tailed T test, n.s.: non significant 
biosynthetic and intermediary metabolism as well as translation (including ribosome biogenesis and protein folding) (Fig 3A). This indicates that changing PK levels triggered metabolic reactions and pathways required for the biosynthesis of proteins (biosynthethic metabolism). Importantly, the list of significantly regulated yeast genes contained several enzymes involved in the synthesis of amino acids. Amino acid concentrations are central for biosynthetic metabolism, important for growth control, and at the same time implicated in cancer metabolism [1, $26,27]$. Therefore, we continued our survey by quantifying 17 amino acid levels using ninhydrin detection/ion exchange chromatography in wild-type as well as the yeast cells with altered Pyk1p and Pyk2 expression levels. Indeed, PK expression had large effects on the amino acid profiles, nine of the 17 profiled amino acids were significantly $(\mathrm{P}<0.05)$ altered by a change in the $\mathrm{PK}$ expression level (Fig 3). Histidine, threonine or lysine, for instance were strongly decreased in cells with low PYK activity (Fig 3B), whereas glutamine and glutamate were detected at higher concentration (Fig 3C). Only eight amino acids, including methionine, glycine or valine did not significantly respond to a change in the activity of PK (Suppl. Table 2). Thus, PK levels influence the expression of proteins involved in biosynthetic metabolism, and cause a strong signature in the amino acid profile.

\section{PKM2- dependent regulation of AAP-S and SHMP in Thyroid Follicular Adenoma}

Both, the enzymatic/ glycolytic functions of PK, and their role in the regulation of redox processes are highly conserved from yeast to humans [7, 8]. Therefore we speculated that also this newly discovered aspect of the PK function could be conserved. Therefore, we selected PK targets for which we could identify a human orthologue, and tested if they were expressed at sufficient level to be quantified by SRM in thyroid follicular adenoma.

Thyroid follicular adenomas are benign human cancers which exhibit clear up-regulation of PKM2 when compared to normal thyroid tissue [20] (Fig 4A for a summary). First, we determined the activity of the five respiratory chain complexes in these samples. The enzymatic activities were normalized to the citrate synthase, which is a marker of mitochondrial mass. A clear reduction in respiratory chain activity (complexes I - IV) was observed in thyroid follicular adenomas compared to normal adjacent thyroid tissue (Fig 4 B). Thus, exhibiting upregulation of PKM2, and low activity of the respiratory chain, follicular thyroid adenomas represented an archetypal tissue exhibiting the Warburg effect.

Using the QTRAP mass spectrometer, we tested if human orthologues of the yeast proteins can be quantified by label free LC-SRM in the trypsin digested cell extracts [25]. We could identify peptides and SRM transitions suitable for robust relative quantification of the aminopeptidase AAP-S and serine hydroxymethyltransferase SHMT1 (Fig 4). Specific SRM signals corresponding to the other three proteins were of too low signal or specificity for a reliable quantification with our method (data not shown). In adenoma, both AAP-S and SHMT1 were present in higher concentration compared to normal thyroid tissue (Fig 4C), showing that these proteins are upregulated in the tumor state. The effect was prominent for AAP-S; the AAP-S levels clearly distinguished normal and adenoma tissue $(\mathrm{p}<$ 0.01). Next, we plotted the relative expression level of AAP-S versus the concentration of PKM2 (Fig 4C, right panel) Both, AAP-S and SHMT1 did not correlate with the expression level of PKM2 in the healthy tissue. However, both proteins showed correlation with the PKM2 level in the tumor state (Fig 4C). AAP-S levels exhibited an almost perfect linear correlation with the PKM2 concentration. Thus, direct orthologues of two yeast PK target genes were upregulated in human thyroid follicular adenomas. Moreover, their expression correlates with the PKM2 level in the tumor, but not in healthy tissue.

\section{DISCUSSION}

Reaction cascades of central metabolic pathways are well characterized, yet still relatively little is known about their regulation and coordination. However, homeostasis of cellular metabolites and metabolic fluxes is essential for cellular growth, and needs to be adapted to the growth conditions of all living cells [3, 28-32] Recently, it has become clear that the metabolic enzyme PK is required for proper coordination of energy- and redox metabolism. In its glycolytic role, PK converts phosphoenolpyruvate (PEP) to pyruvate, yielding a molecule of ATP, and thus, this reaction is required for the net ATP production in glycolysis [33]. Interest in PKM2 had increased markedly after it had been suggested that PKM2 expression in adults could be specific to cancer cells, and that a PKM1 to PKM2 isoform switch might be responsible for increased fermentative metabolism (Warburg effect) of cancer cells $[34,35]$. Cancer specificity of PKM2 could however not be confirmed in recent studies, as PKM2 was found to dominate over PKM1 in most adult tissue, including kidney, lung, liver, thyroid, bladder and colon [20], except however muscle [34]. Despite not specific to cancer, several studies established an essential role of PKM2 in metabolic regulation and the oxidative stress response, processes of high importance for cancer development [17-19, 36-40]. First, the literature appears consistent that PKM2 is upregulated on transcript or protein level in several malignant and benign tumors. These studies included human melanoma [41] childhood leukemia [42], renal cell carcinoma, bladder carcinoma, hepatocellular carcinoma, colorectal carcinoma, lung carcinoma, thyroid follicular adenoma [20], and mouse kidney tumors [36]. 
Several cancer tissues also expressed the PKM1 isoform, however at much lower levels than PKM2. In some but not all tumors, also the PKM1 isoform was found to be upregulated [20].

Conversely, at the same time it has been reported that the PK activity is reduced by posttranslational modifications in cancer cells, and that this reduction is required for their proliferation [18, 19, 43]. Reduced PK activity was attributed to PKM2 phosphorylation [19], thiol oxidation [18], and acetylation [43]. This indicates that PKM2 is upregulated in cancer to increase the protein content, but not to increase the overall PK enzymatic activity. However, it has also to be noted that it is yet unclear to which extent the identified modifications might also be found on PKM2 in normal human tissue, and thus, and as to which extend they are cancer cell specific.

Here, we followed the proteome wide consequences of the Pyk1p downregulation by creating shotgun proteome of yeast expressing Pyk1p at high and low levels using a latest-generation QqTOF proteomics platform, a TripleTOFTM 5600 (AB SCIEX) mass spectrometer coupled to a high-pressure nanoLC system was used for a label free proteome profiling. Using the paragon research engine, we identified $>900$ proteins per single run. A set of 393 proteins met the stringent criteria for being quantified, i.e. that a minimum of three specific peptides were identities in all 19 shotgun runs. The proteome profiles clearly clustered dependent on the PK expression level (Fig 1C) This clear separation was caused by 7.8 percent (31) proteins that were significantly responding to changing PK levels.

These numbers illustrate both the advantages and disadvantages of current label free proteomic approaches. On the plus side, label-free profiling is much more flexible and convenient, as isotope labeled standards are costly, and these standards can only be created for a limited number of species. In this particular case, i.e. SILAC labeling was difficult, as the yeast genetic modifications necessary [44] would impact yeast amino acid transport and metabolism, and thus potentially interfere with the biological function of PK. On the down side, at the current state of the art, the number of proteins quantifiable in a labeled experiments is higher, as the peptide pools can easily be pre-separated before the LC runs. However, also when using isotope labels, the number of replicate injections may be limited by the throughput of the nanoLC gradients (i.e. the 19 injections using at a $3 \mathrm{hr}$ gradient required one week of instrument time). These current bottlenecks for label-free approaches might however improve in the near future, as recent developments in data-independent acquisition (i.e. SWATH [45]) will facilitate significant faster runtime while increasing in the number of quantifiable peptides and proteins.

To verify the proteome profiles, we applied the targeted MS technique selective reaction monitoring (SRM) and analyzed the expression of nine randomly chosen PYK targets. Facilitated by the shorter runtimes and the high reproducibility of the SRM experiments, we included three additional strains that have different PK activities, the wild type parent BY4741, as well as two yeast strains expressing the PYK2 both at high and low level [17]. For seven of the nine tested proteins, this extended study did confirm Pyk1p, and also Pyk2p dependent regulation. Furthermore, the SRM experiments allowed grouping of these PK targets according to their co-expression pattern. Remarkably, co-expressed proteins formed functional clusters: proteins up-regulated were glycolytic enzymes, proteins downregulated were involved in translation. PK dependence of glycolytic enzyme expression is an interesting parallel to the humans in respect to cancer development: hydroxylated PKM2 leads to an upregulation of glycolytic enzymes through interacting with hypoxia inducible factor $-1 \alpha$ (HIF $1 \alpha$ ) $[38,39]$. Thus, in both species regulation of glycolytic enzyme abundances can be attributed to PK, and hence, at least also parts of non-catabolic functions of PK appear to be conserved between yeast and humans. Interestingly, we also observed overlapping targets upon an activation of the PPP during the oxidative stress response [16]. As low PYK activity increases the concentration of PPP metabolites [17], it is conceivable that the PPP is involved in gene expression regulation mediated by PK. In this context is has to be noted that switches of glycolysis to the PPP can howeveronly partly attributed to transcriptional regulation, i.e. metabolic shifts in glycolysis and the PPP can be regulated on the metabolic level only [46], and may occur much faster than transcriptional regulation [47].

The vast majority of identified PK targets were involved in metabolic processes related to glycolysis, the synthesis of nucleic acids, amino acids and proteins. Although it is assumed that PK mediates the regulation of glycolysis [39, 40], similar mechanisms might also exist for amino acid metabolism. To obtain a metabolic picture of the global PK function in this process we thus set out to use ion exchange chromatography and ninhydrin detection to profile the levels of free amino acids in the PK model strains. Comparing yeast with different PK expression level and activity, we found a clear reconfiguration of the overall amino acid profile. In cells with low PK activity, seven of 17 amino acids were significantly less concentrated, and two were higher concentrated.

Amino acid levels are important indicators for the metabolic homeostasis of cells - and closely interconnected with protein metabolism. In yeast, mammalian cells, and flies, proteasome inhibition is lethal due by causing amino acid shortage [48], implying that maintaining sufficient amino acid supply is crucial for proliferating cells and tumors. One of the amino acids we found at decreased concentration was serine. Remarkably, it has recently been described for human cells that PKM2 stimulates de novo serine biosynthesis [49], and that in turn serine can act as allosteric activator of PKM2 [50]. 
This implies a feedback circle involving PYK that controls serine production in mammalian cells, providing evidence that the PK's role in regulation of serine metabolism is conserved between yeast and mammals.

Only two amino acids increased in concentration when there was low PYK activity, glutamine and glutamate. Turnover of these two amino acids may be of crucial importance for cancer metabolism, as cells can obtain a substantial amount of their energy through the process of glutaminolysis $[3,51]$. A change in pyruvate kinase expression seem thus shift energy metabolism towards glutaminolysis, providing sufficient energy supply for cancer cells.

To test for conservation in humans, we searched for human orthologues of the proteins regulated by yeast PK. Remarkably, two direct orthologues were known to be associated with cancer progression. The first protein, asparagine synthase, is the orthologue of yeast protein ASN1, ASNS (Fig 3). ASNS is as potential target for the treatment of castration-resistant stage of prostate cancer [52]. Moreover, the expression level of this enzyme is an indicator for the efficiency of the enzyme drug L-Asparaginase (L-ASP) in ovarian cancer [53]. The second protein, serine hydroxymethylstransferase (SHMT1), is the human orthologue of the yeast protein SHM1. SHMT1 catalyzes the formation of glycine and methylene tetrahydrofolate which are important precursors for nucleotide biosynthesis, and is discussed as a therapeutic target since the late 1980s [54].

Taken together, these observations implicated that the function of PK in regulating amino acid metabolism is conserved between yeast and human. Therefore, we continued our investigations by quantifying further potential PK targets in a human tumor. For two reasons, we selected thyroid follicular adenoma for these investigations: First, these benign cancers are characterized by a clear upregulation of the PKM2 enzyme [20]. Indeed, normal and adenoma biopsies are clearly distinguished by the PKM2 level ([20], graphically illustrated in Fig 4A). Also PKM1 was expressed in these tumor tissues, but at around a 50 fold lower level. In difference to PKM2, $P K M 1$ concentrations did not distinguish healthy thyroid from follicular adenoma tissue. Second, as adenomas are slow growing tumors, one can exclude unspecific effects which may result from altered metabolism of the rapidly proliferating cells. Finally, the activity of the respiratory chain complexes I - IV, determined in both the healthy as well as the adenoma biopsies, indicated significantly lowered activity of mitochondrial metabolism (Fig 4B). Thus, being characterized by increased PKM2 levels and a decrease in respiratory chain efficiency, thyroid follicular adenoma represented tissue exhibiting classic features of the 'Warburg effect'

SRM assays were tested for all five human proteins, but only SHMT1 and the aminopeptidase AAP-S were detected at sufficient intensity for reliable relative quantification by our LC-MS/MS workflow. Remarkably, AAP-S and SHMT1 were both found clearly upregulated in these adenoma samples. At the measure of the relative expression level, AAP-S levels clearly distinguished adenoma and that of control tissue $(p<0.01)$. Plotted against the absolute concentration of PKM2, both targets created clusters that distinguished the adenomas from the control tissue. Thus, expression of two direct protein orthologues to the yeast PK targets correlate with PKM2 expression in thyroid follicular adenoma.

\section{CONCLUSIONS}

Pyruvate kinase emerges as a central hub in the regulation of metabolic pathways important for cell proliferation and cancer. As a glycolytic enzyme, it is responsible for the net ATP yield of glycolysis, and the control of the redox state. Here, a combined analysis of label-free shotgun proteomics, targeted proteomics and amino acid profiling reveals that this protein is further important for the coordination of biosynthesis of an orchestra of amino acids. Glutamine and glutamate levels were highly increased in cells with low PK expression levels, which seem to connect the process of glutaminolysis with the regulation of carbon metabolism. Seven of the remaining amino acids were decreased in concentration, including serine, which has recently been reported to be a direct regulator of PKM2 activity in humans [49, 50]. Finally, studies in thyroid follicular adenoma indicated that part of this PK function is conserved in man, and revealed two protein biomarkers of this disease state. AAP-S and SHMT1 correlated with the PKM2 expression level and were upregulated in the tumor. Thus, PK plays an evolutionary conserved role in the coordination of carbon and amino acid homeostasis, making it and its downstream effectors promising candidates for modulating (cancer) metabolism for therapeutic purposes.

\section{METHODS}

\section{Yeast strains and growth conditions}

$\Delta p y k 1 \Delta p y k 2$ yeast strains expressing Pyk1p or Pyk2p under control of the TEF1 or CYC1 promoter were described previously [17]. The $\Delta p y k 2$ strain was generated from BY4741 by single gene replacement with kanMX4, and verified by PCR and SRM. Yeast was grown in yeast peptone (YP) media containing 2\% dextrose, 2\% galactose, $3 \%$ ethanol $/ 0.1 \%$ glucose or $2 \%$ raffinose as indicated. All measurements were performed on mid-log batch cultures. 


\section{Enzyme assays}

Follicular thyroid adenoma and normal adjacent thyroid tissues (20-100 $\mathrm{mg}$ ) were homogenized with a tissue disintegrator (Ultraturrax, IKA, Staufen, Germany) in extraction buffer ( $20 \mathrm{mM}$ Tris- $\mathrm{HCl}, \mathrm{pH}$ 7.6, $250 \mathrm{mM}$ sucrose, $40 \mathrm{mM} \mathrm{KCl}, 2 \mathrm{mM}$ EGTA) and finally homogenized with a motor-driven Teflon-glass homogenizer (Potter S, Braun, Melsungen, Germany). The homogenate was centrifuged at $600 \mathrm{~g}$ for $10 \mathrm{~min}$ at $4^{\circ} \mathrm{C}$. The supernatant $(600 \mathrm{~g}$ homogenate) containing the mitochondrial fraction was used for measurement of enzyme activities. OXPHOS enzyme and citrate synthase activity [55-59] were measured as described earlier. Activity of respiratory chain complexes is given relative to citrate synthase activity.

\section{Shotgun proteomics}

An TripleTOFTM 5600 system (AB SCIEX, Concord, Ontario, Canada) was coupled with a Dionex Ultimate 3000 RSLCnano system (Dionex, Sunnyvale, California). Details to instrumental parameters and data processing can be found in the supplementary material. Briefly the raw data was analyzed using ProteinPilot ${ }^{\mathrm{TM}}$ Software v.4.0 (AB SCIEX) on database UniprotKB/ Swiss-Prot 57.15 in thorough search mode applying the integrated false discovery rate analysis tool (PSPEP) [60].

Subsequent quantification of peptides and proteins was performed automatically in PeakView ${ }^{\mathrm{TM}}$ Software for proteins with $<1 \%$ false discovery rate as performed using PSPEP. Areas of peptides and proteins were automatically transferred to MarkerView ${ }^{\mathrm{TM}}$ Software for visualization of differences for technical replicates and statistical analysis using principal component analysis followed by principal component variable grouping to identify protein groups with similar trends in regulation.

\section{Selective reaction monitoring}

(SRM) analysis was performed on a QTRAP ${ }^{\circledR} 5500$ hybrid triple quadrupole/ ion trap mass spectrometer instrument (AB SCIEX, Concord, ON, Canada) coupled to an Eksigent 2D ultra nano LC system (Eksigent, CA, USA) as described earlier [20, 25]. In brief, SRM assay development for the target proteins was developed using the MRMPilot ${ }^{\mathrm{TM}}$ software (AB SCIEX) version 2.1. Identity of peptides was confirmed using the MIDAS ${ }^{\text {TM }}$ workflow (MRM Initiated Detection And Sequencing) $[25,61]$. Quantification of the potential PYK targets was achieved by normalization to cellular reference proteins [25] V-type proton ATPase catalytic subunit A (V-ATPase) and actin. The ratios of their SRM intensities were similar in all samples investigated. SRM transitions and collision energy (CE) settings are given in the Suppl. Table 3.

\section{Amino acid Analysis}

Amino acid analysis was performed using ionexchange chromatography on a Biochrom 30 amino acid analyzer with ninhydrin detection. Each sample was analyzed in triplicate. $200 \mu 1$ sample was added to 200 $\mu \mathrm{l}$ of $0.5 \mathrm{mM}$ DL-2,4-diamino-n-butyratedihydrochloride (used as internal standard) and $4.9 \% 5$-sulfosalicylic acid to precipitate the protein. Samples were mixed and centrifuged for 10 minutes at $3345 \mathrm{~g}$ and the supernatant was filtered (Ultrafree-MC Durapore PVDF, $0.22 \mu \mathrm{m}$; Waters, UK) for 10 minutes at $3345 \mathrm{~g}$ to remove all protein precipitate. A series of lithium citrate buffer solutions were run through a lithium column $(8 \mu \mathrm{m}$ Biochrom, UK) containing the samples. Individual amino acids were eluted according to their $\mathrm{pH}$. Reaction with ninhydrin was utilized to elicit a spectrum of colors at different wavelengths giving a chromatogram representing the concentrations of each amino acid.

\section{ETHICS}

Four thyroid follicular adenomas and adjacent normal appearing thyroid tissue from patients were obtained from the Institute of Pathology, University Hospital Salzburg, Austria.

The study was performed according to the Austrian Gene Technology Act. Experiments were performed in accordance with the Helsinki declaration of 1975 (revised 2000) and the guidelines of the Salzburg State Ethics Research Committee as not being a clinical drug trial nor epidemiological investigation. Furthermore, the study did not extend to examination of individual case records. The anonymity of the patients has been ensured.

\section{AUTHOR CONTRIBUTIONS}

KB, MG, NMG, RF, AK, MW performed experiments, ST programmed software, DN, BK, obtained and verified human tissue, MR, MG, KB analyzed data, MR wrote the first manuscript draft, MR, KB, BK, HL finalized the manuscript

\section{ACKNOWLEDGEMENTS}

We thank Prof. Cornelis Jakoks (VUMC Amsterdam) for critical discussions and support of the study, and Erik Wever and Abdallative Bakkali (both VUMC) for their help with the amino acid analysis. We acknowledge funding from the Max Planck Society, the Wellcome Trust (RG 093735/Z/10/Z) and the ERC (Starting grant 260809). M.R. is a Wellcome Trust Research Career Development and Wellcome-Beit prize 
fellow.

\section{REFERENCE}

1. Cairns RA, Harris IS and Mak TW. Regulation of cancer cell metabolism. Nature reviews. 2011; 11(2):85-95.

2. Hanahan D and Weinberg RA. Hallmarks of cancer: the next generation. Cell. 2011; 144(5):646-674.

3. Israel M and Schwartz L. The metabolic advantage of tumor cells. Molecular cancer. 2011; 10:70.

4. Guido C, Whitaker-Menezes D, Lin Z, Pestell RG, Howell A, Zimmers TA, Casimiro MC, Aquila S, Ando S, Martinez-Outschoorn UE, Sotgia F and Lisanti MP. Mitochondrial fission induces glycolytic reprogramming in cancer-associated myofibroblasts, driving stromal lactate production, and early tumor growth. Oncotarget. 2012; 3(8):798-810.

5. Warburg O. Origin of Cancer Cells. Science (New York, NY. 1956; 123(3191):309-314.

6. Bayley JP and Devilee P. The Warburg effect in 2012. Current opinion in oncology. 2011; 24(1):62-67.

7. Hamanaka RB and Chandel NS. Cell biology. Warburg effect and redox balance. Science (New York, NY. 2011; 334(6060):1219-1220.

8. Grüning N-M and Ralser M. Cancer: Sacrifice for survival. Nature. 2011; 480(7376):190-191.

9. Pelicano H, Carney D and Huang P. ROS stress in cancer cells and therapeutic implications. Drug Resist Updat. 2004; 7(2):97-110.

10. Perera RM and Bardeesy N. Cancer: when antioxidants are bad. Nature. 2011; 475(7354):43-44.

11. Apel $\mathrm{K}$ and Hirt H. Reactive oxygen species: metabolism, oxidative stress, and signal transduction. Annual review of plant biology. 2004; 55:373-399.

12. Cadenas E and Davies KJ. Mitochondrial free radical generation, oxidative stress, and aging. Free radical biology \& medicine. 2000; 29(3-4):222-230.

13. Ralser M, Wamelink MM, Kowald A, Gerisch B, Heeren G, Struys EA, Klipp E, Jakobs C, Breitenbach M, Lehrach $\mathrm{H}$ and Krobitsch S. Dynamic rerouting of the carbohydrate flux is key to counteracting oxidative stress. J Biol. 2007; 6(4):10.

14. Ying $\mathrm{W}$. NAD $+/ \mathrm{NADH}$ and $\mathrm{NADP}+/ \mathrm{NADPH}$ in cellular functions and cell death: regulation and biological consequences. Antioxid Redox Signal. 2008; 10(2):179206.

15. Pollak N, Dolle C and Ziegler M. The power to reduce: pyridine nucleotides--small molecules with a multitude of functions. Biochem J. 2007; 402(2):205-218.

16. Kruger A, Gruning NM, Wamelink MM, Kerick M, Kirpy A, Parkhomchuk D, Bluemlein K, Schweiger MR, Soldatov A, Lehrach H, Jakobs C and Ralser M. The pentose phosphate pathway is a metabolic redox sensor and regulates transcription during the antioxidant response. Antioxid Redox Signal. 2011; 15(2):311-324.

17. Gruning NM, Rinnerthaler M, Bluemlein K, Mulleder M, Wamelink MM, Lehrach H, Jakobs C, Breitenbach M and Ralser M. Pyruvate kinase triggers a metabolic feedback loop that controls redox metabolism in respiring cells. Cell metabolism. 2011; 14(3):415-427.

18. Anastasiou D, Poulogiannis G, Asara JM, Boxer MB, Jiang JK, Shen M, Bellinger G, Sasaki AT, Locasale JW, Auld DS, Thomas CJ, Vander Heiden MG and Cantley LC. Inhibition of pyruvate kinase M2 by reactive oxygen species contributes to cellular antioxidant responses. Science (New York, NY. 2011; 334(6060):1278-1283.

19. Hitosugi T, Kang S, Vander Heiden MG, Chung TW, Elf S, Lythgoe K, Dong S, Lonial S, Wang X, Chen GZ, Xie J, Gu TL, Polakiewicz RD, Roesel JL, Boggon TJ, Khuri FR, et al. Tyrosine phosphorylation inhibits PKM2 to promote the Warburg effect and tumor growth. Sci Signal. 2009; 2(97):ra73.

20. Bluemlein K, Gruning NM, Feichtinger RG, Lehrach H, Kofler B and Ralser M. No evidence for a shift in pyruvate kinase PKM1 to PKM2 expression during tumorigenesis. Oncotarget. 2011; 2(5):393-400.

21. Boles E, Schulte F, Miosga T, Freidel K, Schluter E, Zimmermann FK, Hollenberg CP and Heinisch JJ. Characterization of a glucose-repressed pyruvate kinase (Pyk2p) in Saccharomyces cerevisiae that is catalytically insensitive to fructose-1,6-bisphosphate. Journal of bacteriology. 1997; 179(9):2987-2993.

22. Andrews GL, Simons BL, Young JB, Hawkridge AM and Muddiman DC. Performance characteristics of a new hybrid quadrupole time-of-flight tandem mass spectrometer (TripleTOF 5600). Analytical chemistry. 2011; 83(13):5442-5446.

23. Bisson N, James DA, Ivosev G, Tate SA, Bonner R, Taylor $\mathrm{L}$ and Pawson T. Selected reaction monitoring mass spectrometry reveals the dynamics of signaling through the GRB2 adaptor. Nat Biotechnol. 2011; 29(7):653-658.

24. Gallien S, Duriez E and Domon B. Selected reaction monitoring applied to proteomics. J Mass Spectrom. 2011; 46(3):298-312.

25. Bluemlein $\mathrm{K}$ and Ralser M. Monitoring protein expression in whole-cell extracts by targeted label- and standard-free LC-MS/MS. Nature Protocols. 2011; 6(6).

26. Eng $\mathrm{CH}$ and Abraham RT. The autophagy conundrum in cancer: influence of tumorigenic metabolic reprogramming. Oncogene. 2011; 30(47):4687-4696.

27. Kim $J$ and Guan KL. Amino acid signaling in TOR activation. Annual review of biochemistry. 2011; 80:10011032.

28. Grüning NM, Lehrach $\mathrm{H}$ and Ralser M. Regulatory crosstalk of the metabolic network. Trends Biochem Sci. 2010; 35(4):220-227.

29. Heinemann M and Sauer U. Systems biology of microbial 
metabolism. Current opinion in microbiology. 2010; 13(3):337-343.

30. Wolf A, Agnihotri S and Guha A. Targeting metabolic remodeling in glioblastoma multiforme. Oncotarget. 2010; 1(7):552-562.

31. Pavlides S, Tsirigos A, Vera I, Flomenberg N, Frank PG, Casimiro MC, Wang C, Pestell RG, Martinez-Outschoorn UE, Howell A, Sotgia F and Lisanti MP. Transcriptional evidence for the "Reverse Warburg Effect" in human breast cancer tumor stroma and metastasis: similarities with oxidative stress, inflammation, Alzheimer's disease, and "Neuron-Glia Metabolic Coupling". Aging. 2010; 2(4):185199.

32. Demaria M, Giorgi C, Lebiedzinska M, Esposito G, D’Angeli L, Bartoli A, Gough DJ, Turkson J, Levy DE, Watson CJ, Wieckowski MR, Provero P, Pinton P and Poli V. A STAT3-mediated metabolic switch is involved in tumour transformation and STAT3 addiction. Aging. 2010; 2(11):823-842.

33. Kubowitz F and Ott P. Isolierung von Gärungsfermenten aus menschlichen Muskeln. . Biochem Z. 1944; 317:193203.

34. Christofk HR, Vander Heiden MG, Harris MH, Ramanathan A, Gerszten RE, Wei R, Fleming MD, Schreiber SL and Cantley LC. The M2 splice isoform of pyruvate kinase is important for cancer metabolism and tumour growth. Nature. 2008; 452(7184):230-233.

35. Mazurek S, Boschek CB, Hugo F and Eigenbrodt E. Pyruvate kinase type M2 and its role in tumor growth and spreading. Semin Cancer Biol. 2005; 15(4):300-308.

36. Sun Q, Chen X, Ma J, Peng H, Wang F, Zha X, Wang Y, Jing Y, Yang H, Chen R, Chang L, Zhang Y, Goto J, Onda H, Chen T, Wang MR, et al. Mammalian target of rapamycin up-regulation of pyruvate kinase isoenzyme type M2 is critical for aerobic glycolysis and tumor growth. Proc Natl Acad Sci U S A. 2011; 108(10):4129-4134.

37. Vander Heiden MG, Locasale JW, Swanson KD, Sharfi H, Heffron GJ, Amador-Noguez D, Christofk HR, Wagner G, Rabinowitz JD, Asara JM and Cantley LC. Evidence for an alternative glycolytic pathway in rapidly proliferating cells. Science (New York, NY. 2010; 329(5998):1492-1499.

38. Luo W, Hu H, Chang R, Zhong J, Knabel M, O'Meally R, Cole RN, Pandey A and Semenza GL. Pyruvate kinase M2 is a PHD3-stimulated coactivator for hypoxia-inducible factor 1. Cell. 2011; 145(5):732-744.

39. Luo W and Semenza GL. Pyruvate kinase M2 regulates glucose metabolism by functioning as a coactivator for hypoxia-inducible factor 1 in cancer cells. Oncotarget. 2011; 2(7):551-556.

40. Chaneton B and Gottlieb E. Rocking cell metabolism: revised functions of the key glycolytic regulator PKM2 in cancer. Trends Biochem Sci. 2012; 37(8):309-316.

41. Huang SK, Darfler MM, Nicholl MB, You J, Bemis KG, Tegeler TJ, Wang M, Wery JP, Chong KK, Nguyen L,
Scolyer RA and Hoon DS. LC/MS-based quantitative proteomic analysis of paraffin-embedded archival melanomas reveals potential proteomic biomarkers associated with metastasis. PLoS One. 2009; 4(2):e4430.

42. Hulleman E, Broekhuis MJ, Pieters R and Den Boer ML. Pyruvate kinase M2 and prednisolone resistance in acute lymphoblastic leukemia. Haematologica. 2009; 94(9):13221324.

43. Lv L, Li D, Zhao D, Lin R, Chu Y, Zhang H, Zha Z, Liu Y, Li Z, Xu Y, Wang G, Huang Y, Xiong Y, Guan KL and Lei QY. Acetylation targets the M2 isoform of pyruvate kinase for degradation through chaperone-mediated autophagy and promotes tumor growth. Mol Cell. 2011; 42(6):719-730.

44. de Godoy LM, Olsen JV, de Souza GA, Li G, Mortensen $\mathrm{P}$ and Mann M. Status of complete proteome analysis by mass spectrometry: SILAC labeled yeast as a model system. Genome biology. 2006; 7(6):R50.

45. Gillet LC, Navarro P, Tate S, Roest H, Selevsek N, Reiter L, Bonner R and Aebersold R. Targeted data extraction of the MS/MS spectra generated by data independent acquisition: a new concept for consistent and accurate proteome analysis. Mol Cell Proteomics. 2012.

46. Daran-Lapujade P, Rossell S, van Gulik WM, Luttik MA, de Groot MJ, Slijper M, Heck AJ, Daran JM, de Winde JH, Westerhoff HV, Pronk JT and Bakker BM. The fluxes through glycolytic enzymes in Saccharomyces cerevisiae are predominantly regulated at posttranscriptional levels. Proc Natl Acad Sci U S A. 2007; 104(40):15753-15758.

47. Ralser M, Wamelink MM, Latkolik S, Jansen EE, Lehrach $\mathrm{H}$ and Jakobs C. Metabolic reconfiguration precedes transcriptional regulation in the antioxidant response. Nat Biotechnol. 2009; 27(7):604-605.

48. Suraweera A, Munch C, Hanssum A and Bertolotti A. Failure of Amino Acid Homeostasis Causes Cell Death following Proteasome Inhibition. Molecular cell. 2012.

49. Ye J, Mancuso A, Tong X, Ward PS, Fan J, Rabinowitz JD and Thompson CB. Pyruvate kinase M2 promotes de novo serine synthesis to sustain mTORC1 activity and cell proliferation. Proc Natl Acad Sci U S A. 2012; 109(18):6904-6909.

50. Chaneton B, Hillmann P, Zheng L, Martin AC, Maddocks OD, Chokkathukalam A, Coyle JE, Jankevics A, Holding FP, Vousden KH, Frezza C, O'Reilly M and Gottlieb E. Serine is a natural ligand and allosteric activator of pyruvate kinase M2. Nature. 2012.

51. Medina MA and Nunez de Castro I. Glutaminolysis and glycolysis interactions in proliferant cells. The International journal of biochemistry. 1990; 22(7):681-683.

52. Sircar K, Huang H, Hu L, Cogdell D, Dhillon J, Tzelepi V, Efstathiou E, Koumakpayi IH, Saad F, Luo D, Bismar TA, Aparicio A, Troncoso P, Navone $\mathrm{N}$ and Zhang W. Integrative molecular profiling reveals asparagine synthetase is a target in castration-resistant prostate cancer. The American journal of pathology. 2012; 180(3):895-903. 
53. Lorenzi PL and Weinstein JN. Asparagine synthetase: a new potential biomarker in ovarian cancer. Drug news \& perspectives. 2009; 22(1):61-64.

54. Snell K, Natsumeda Y, Eble JN, Glover JL and Weber G. Enzymic imbalance in serine metabolism in human colon carcinoma and rat sarcoma. British journal of cancer. 1988; 57(1):87-90.

55. Feichtinger RG, Zimmermann FA, Mayr JA, Neureiter D, Ratschek M, Jones N, Sperl W and Kofler B. Alterations of respiratory chain complexes in sporadic pheochromocytoma. Frontiers in bioscience (Elite edition). 2011; 3:194-200.

56. Feichtinger RG, Neureiter D, Royer-Pokora B, Mayr JA, Zimmermann FA, Jones N, Koegler C, Ratschek M, Sperl $\mathrm{W}$ and Kofler B. Heterogeneity of mitochondrial energy metabolism in classical triphasic Wilms' tumor. Frontiers in bioscience (Elite edition). 2011; 3:187-193.

57. Feichtinger RG, Neureiter D, Mayr JA, Zimmermann FA, Berthold F, Jones N, Sperl W and Kofler B. Loss of mitochondria in ganglioneuromas. Frontiers in bioscience (Elite edition). 2011; 3:179-186.

58. Feichtinger RG, Zimmermann F, Mayr JA, Neureiter D, Hauser-Kronberger C, Schilling FH, Jones N, Sperl W and Kofler B. Low aerobic mitochondrial energy metabolism in poorly- or undifferentiated neuroblastoma. BMC cancer. 2010; 10:149.

59. Mayr JA, Meierhofer D, Zimmermann F, Feichtinger R, Kogler C, Ratschek M, Schmeller N, Sperl W and Kofler B. Loss of complex I due to mitochondrial DNA mutations in renal oncocytoma. Clin Cancer Res. 2008; 14(8):22702275.

60. Tang WH, Shilov IV and Seymour SL. Nonlinear fitting method for determining local false discovery rates from decoy database searches. Journal of proteome research. 2008; 7(9):3661-3667.

61. Unwin RD, Griffiths JR and Whetton AD. A sensitive mass spectrometric method for hypothesis-driven detection of peptide post-translational modifications: multiple reaction monitoring-initiated detection and sequencing (MIDAS). Nat Protoc. 2009; 4(6):870-877. 